\title{
Endodontic Therapy of Maxillary Third Molar with Supernumerary Root with Four Root Canals-A Rare Case
}

\author{
P Vishnu Unni ${ }^{1}$ and M Kundabala ${ }^{2 *}$ \\ ${ }^{1}$ Former, post graduate student of certificate course in Restorative dentistry, India \\ ${ }^{2}$ Professor, Department of Conservative Dentistry and Endodontics, India
}

Received: August 21, 2017; Published: September 07, 2017

*Corresponding author: M Kundabala, Professor, Department of Conservative Dentistry and Endodontics, Mangalore, Manipal University,

Mangaluru-575001, Karnataka, India, Email: kundakamath@gmail.com/kunda.kamath@manipal.edu

\begin{abstract}
Success of endodontic therapy is multi factorial. Starting from good case selection, proper treatment, thorough cleaning and shaping, fluid tight obturation and good seal achieved by post endodontic restoration. One of the reasons for failure of root canal therapy is complex internal anatomy of the teeth which is non-negotiable through orthograde approach. Thorough knowledge of the internal anatomy and its variations is very important to provide the best treatment for the patient. Clinician has to face challenges if there are fused canals, calcified canals, Radix entomolaris, taurodontism and most commonly presence of additional canals. Maxillary third molar is one such tooth where root canal therapy is very difficult to perform not only because of its location in the arch but also due to its varied internal anatomy. The present paper describes variation in the internal anatomy of a maxillary 3rd molar with additional canal and also the importance of magnification in endodontic.
\end{abstract}

Keywords: Additional canals; Maxillary third molar; Internal anatomy; Dental loupes

\section{Introduction}

The presence of a missed canal in the course of root canal treatment is one of the common reasons for failure of therapy. The consequence of a missed canal will or may lead to chronic infection and post operative flare ups. Hence, it is very important to explore the morphology of the tooth as well its internal anatomy to overcome failure in endodontic practice. Root canal therapy of maxillary third molars is usually indicated when in teeth with strategic reasons such as:

a. An abutment tooth for fabricating a fixed prosthesis when other molar teeth are missing.

b. Presence of posterior teeth in the opposing arch, thus enhancing mastication.

c. Strategic value of tooth for placement of fixed partial denture, when the second molar is missing

There are numerous variations in morphology and anatomy of maxillary third molars. Number of roots and no. of canals present in maxillary $3^{\text {rd }}$ molars usually range from 1-6 [1]. Around $25.8 \%$ of maxillary third molars have three separate roots of which only $10 \%$ had four canals. Roots with 4 canals were found in only $3.2 \%$ of cases. There were fused roots in $38 \%$ of the cases. Five independent roots with 5 canals were found in only $0.6 \% \%$ of the cases [2]. In the present case, there is presence of additional mesiopalatal canal (4th canal) in an additional supernumerary root which is extremely rare in the case of a maxillary third molar. Magnifying dental loupes (2.5 X magnification) were used for visualizing additional canal orifice and also for convenience during the endodontic access cavity preparation.

\section{Case Report}

Sixty three year old male patient reported to the Department of Conservative Dentistry and Endodontic with a chief complaint of lingering pain upon consumption of hot or cold food items. The patient reported within crease in intensity of pain during the night and no relief upon taking analgesics. Patient's medical history was non-contributory. The patient also reported to have been reduced the tooth from occlusion for the relief from pain 2 years from local dentist prior to the present dental visit.

Clinical findings revealed a fractured silver amalgam restoration with evidence of secondary caries with respect to left maxillary third molar. IOPA radiograph in relation to the concerned tooth showed coronal radio-opacity approaching the pulp which was suggestive of deep silver amalgam restoration with secondary caries around it, may have been a causative factor for pupil inflammation. Thermal and electric pulp testing showed exaggerated response with 
lingering pain suggestive of sub acute inflammatory changes in the pulp. A diagnosis of sub acute pulpalgia was made in relation to the maxillary left third molar. Treatment planning included root canal therapy due to the strategic location of the teeth.

After administration of local anesthesia using posterior superior alveolar nerve block with lignocaine hydrochloride $2 \%$ and 1:80,000 adrenalines, defective silver amalgam restoration was removed using a large round bur at high speed under air and water coolant. Secondary caries was excavated by sharp spoon excavator followed by which access opening was initiated. After the tooth was anaesthetized, access cavity was prepared using Endo Z bur (Dentsply Maillefer). Coving of the access in mesiopalatal direction was done to explore the dentinal map. Under indirect vision, three canal orifices - mesiobuccal, distobuccal and palatal were found. A small bleeding point was also noticed slightly mesial and palatal to the mesiobuccal canal orifice which was initially assumed to be a minor perforation.

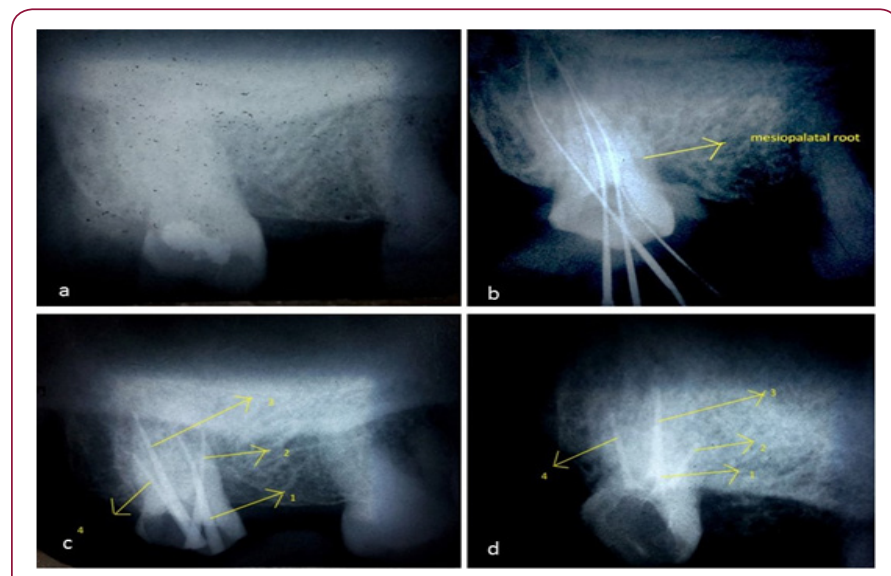

Figure 1: a. Pre-operative IOPA radiograph showing coronal radio-opacity suggestive of dental restoration approaching the pulp, $\mathrm{b}$. Working length radiograph showing the missed mesiopalatal root, c. Master cone radiograph 1-mesiobuccal,2-mesiopalatal,3-palatal,4distobuccal, d. Post obturation radiograph.

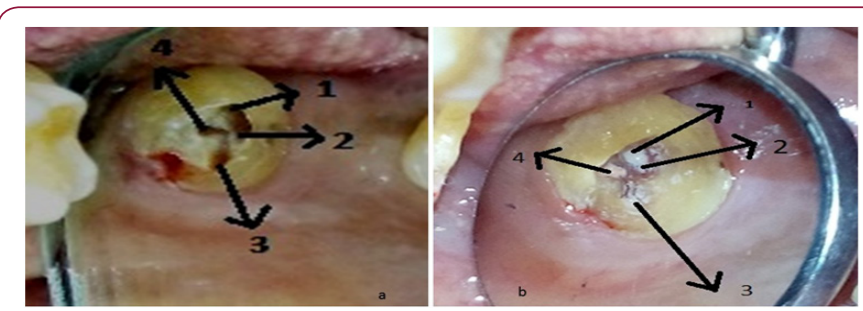

Figure 2: a. Access opening done and cavity preparation modified, b. Post obturation image 1-mesiobuccal,

2-mesiopalatal, 3-palatal and 4-distobuccal.

Tooth was isolated using rubber dam. The three canal orifices were apically enlarged up to a size $25 \mathrm{~K}$ file (Mani, Japan). After achieving a glide path, canal orifices were enlarged using a hand ProTaper SX file (Dentsply Maillefer). The canals were thoroughly irrigated with $5.25 \%$ sodium hypochlorite and EDTA. Working length was determined by IOPA radiograph using Ingle's method. Radiograph showed unusual morphology of supernumerary root and also presence of a fourth canal (Figure 1). With the help of magnification loupes of 2.5X magnification (Heine, USA Ltd. Dover, NH 03820) Resolution Binocular Loupe), the fourth canal orifice was found (Figures 2A \& 2B). Working length of canal radiograph was determined. In the next appointment, canal instrumentation was completed using Pro Taper hand file and rotary files (Dentsply Maillefer) up to F3. The instrumentation was accompanied with the use of root canal lubricant RC-Prep (Premier TM USA) along with $5.25 \%$ sodium hypochlorite and saline. After the cleaning and shaping, closed dressing was given with zinc oxide eugenol temporary restoration.

On the third appointment, under rubber dam isolation the canals were thoroughly irrigated with saline and 5.25\% sodium hypochlorite and the patency of the canals were rechecked. Master cones were selected using radiograph. Canals were finally irrigated with EDTA for one minute followed by $5.25 \%$ sodium hypochlorite which was agitated in the canal using an ISO number $10 \mathrm{~K}$ file (Mani, Japan). The canals were dried using sterile paper points and coated with AH Plus sealer (Dent Spy De Trey) using lentulo spirals (DENCO, Guangdong, China, Mainland). The guttaperchacones were also coated with AH-plus sealer. The canals were obdurate using combination of horizontal and vertical compaction technique and temporized with modified $\mathrm{ZnOE}$ cement. Finally access cavity restoration had done using light cure composite resin, Filtek ${ }^{\mathrm{TM}} \mathrm{Z} 350$ XT Universal (3M, India).

\section{Discussion}

Maxillary or mandibular third molars are usually treated carefully mostly due to their complex morphology and internal anatomy, alternatively to extraction. Due to strategic reasons, this tooth might have to be saved. Maxillary third molar usually has complex internal anatomy. It can show different or bizarre root canal anatomy and hence one must have a thorough knowledge of the internal anatomy. Variations in the number of roots/root canals are more.

Usually for maxillary $3^{\text {rd }}$ molars roots range from one to six. There can be single, double, and three-rooted and four variants either separate or fused encasing one to four root canals [1]. Very few studies have examined the occurrence of the MB2 canal-MB2 in mesiobuccal root- 3.7\% [2]. Stropko evaluated endodontic treatment of twenty third molar teeth in which he found that $20 \%$ study subjects have a second mesiobuccal canal in which all of them were joined and ended in a single foramen [3]. A study by Guerisoli et al. [4] found that $14.2 \%$ of maxillary third molars had a fourth canal and in $1.9 \%$ cases, a fifth canal was seen. Another study by Sidow SJ et al. [5], studied the root canal morphology of maxillary and mandibular third molars and found the following $15 \%$ of the cases had one root, $32 \%$ of the cases had two roots, $45 \%$ of the cases had three roots, $7 \%$ of the cases had four roots. Teeth with a single root showed the most unusual morphology and the number of canals ranged from one to six.

Evaluation of the anatomy of 370 maxillary molars reported the presence of three canals in $75 \%$ of the first molars, $58 \%$ of the second molars and $68 \%$ of the third molars. Four canals were 
located in $25 \%, 42 \%$, and $32 \%$ of the maxillary first, second and third molars respectively [6]. In the majority of cases, mesiobuccal roots have two canals [7]. The incidence of a second mesiobuccal canal varies between $18 \%$ and $96.1 \%$ [8-10]. Four rooted maxillary molar teeth have been reported to occur in $0.9 \%, 1.4 \%$ and up to $7 \%$ in first, second and third maxillary molar teeth respectively $[11,12]$. Literature has shown variant comprises of bifurcation of the mesiobuccal root into two mesial roots (mesiobuccal and mesiopalatal roots). The mesiopalatal root is usually located between the mesiobuccal and palatal root. Typically it has a single root canal although it has been reported to have two root canals with a single apical foramen [13].

In modern root canal therapy, advent of loupes and surgical operating microscopes, magnification helps to identify any variations in the root canal anatomy and have become a basic part of armamentarium in modern endodontic practice. Missing canals lead to subsequent treatment failure. Preoperative CBCT, if available can be used for diagnosis, treatment planning, pretreatment evaluation of internal anatomy and working length determination in endodontic especially in case doubts arise in diagnosis.

\section{Conclusion}

The clinical experience, knowledge and skills of operator regarding internal anatomy, diagnosis along with successful access cavity preparation relies on a sound knowledge of the internal and external morphology of teeth. The use of magnification with illumination using specialized equipment such as surgical loupes with LED lights, surgical operating microscopes and ultrasonic endodontic tips, greatly improve the ability of an operator to identify the orifices of root canals, especially in molar teeth. Missing canals because of poor access cavity design could lead to inadequate cleaning, shaping and obturation and ultimately failure of root canal therapy.

\section{References}

1. Ahmed HM, Abbott PV (2012) Accessory roots in maxillary molar teeth: a review and endodontic considerations. Aust Dent J 57(2): 123-131.

2. Danilo M Zanello Guerisoli, Rusiel Amaro de Souza, Manoel D de Sousa Neto, Ricardo Gariba Silva, Jesus Djalma Pecora (1998) External and Internal Anatomy of Third Molars. Braz Dent J 9(2): 91-94.

3. Hany Mohamed Aly Ahmed (2012) Management of third molar from an endodontic perspective. review article Eur J Dent 1(3): 148-160.

4. Greene D (1973) double canals in single roots. oral surg oral med oral pathol 35(5): 689-696.

5. Strepko JJ (1999) canal morphology of maxillary molars,clinical observations of canal configuration. J Endod 25(6): 446-450.

6. Guerisoli, DeSouza, De Sousa Neto, Silva, Pecora, et al. (1998) External and internal anatomy of third molars. Braz Dent J 9(2): 91-94.

7. SidowSJ, WestLA, Liewehr FR, LoushineRJ (2000) Root Canal morphology of human maxillary and mandibular third molars. J Endod 26(11): 675-678.

8. Mittal N, MalikR (2016) Endodontic management of variations in root canal configuration of maxillary first molar:two case reports. Endodontology 28(1): 68-71.

9. PecoraJD, Woefel JB, Sousa Neto MD, Issa EP (1992) morphologic study of the maxillary molars part II: Internal nantomy. Braz Dent j 3(1): 5357.

10. Hartwell G, Appelstein CM, Lyons WW, Guzek ME (2007) The incidence of four canals in maxillary first molar: clinical determination. J Am Dent Assoc 138(10): 1344-1346.

11. Hartwell G, Bellizi R (1982) clinical investigation of in vivo endodontically treated mandibular and maxillary molars. J Endod 8(12): 555-557.

12. Kulild JC, Peters DD (1990) incidence and configuration of canal systems in the mesiobuccal root of maxillary first and second molars. J Endod 16(7): 311-317.

13. NeelakantanP, SubbaraoC, AhujaR, subbaraoCV, Gutmann JL (2010) CBCT study of root and canal morphology of maxillary first and second molars in Indian population. J Endod 36(10): 1622-1627.

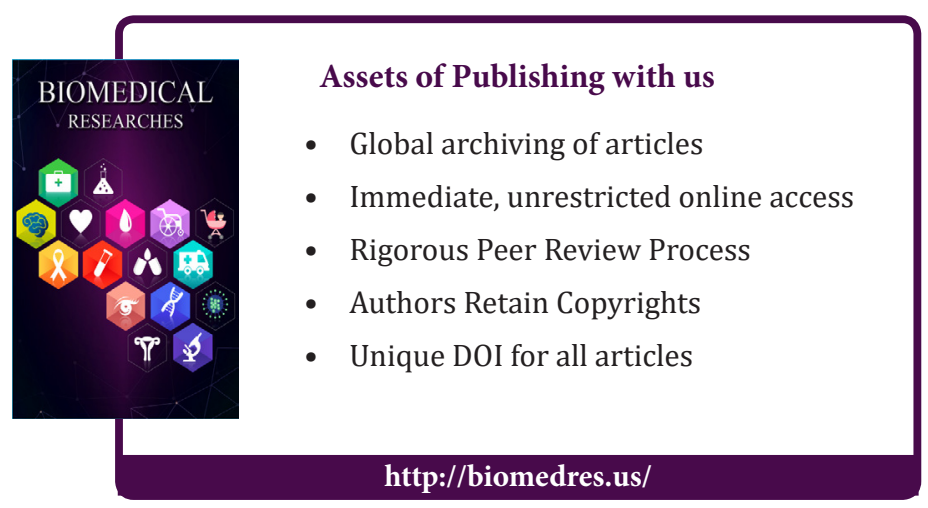

